\title{
A Multi-Objective Genetic Algorithm for Robust Design Optimization
}

\author{
Mian Li \\ Graduate Research Assistant \\ Department of Mechanical \\ Engineering \\ University of Maryland \\ College Park, MD 20742 \\ Tel. +1 3014054919 \\ E-mail:mli6@umd.edu
}

\author{
Shapour Azarm \\ Professor \\ Department of Mechanical \\ Engineering \\ University of Maryland \\ College Park, MD 20742 \\ Tel. +1 3014055250 \\ E-mail: azarm@umd.edu
}

\author{
Vikrant Aute \\ Faculty Research Assistant \\ Department of Mechanical \\ Engineering \\ University of Maryland \\ College Park, MD 20742 \\ Tel. +13014058726 \\ E-mail: vikrant@umd.edu
}

\begin{abstract}
Real-world multi-objective engineering design optimization problems often have parameters with uncontrollable variations. The aim of solving such problems is to obtain solutions that in terms of objectives and feasibility are as good as possible and at the same time are least sensitive to the parameter variations. Such solutions are said to be robust optimum solutions. In order to investigate the trade-off between the performance and robustness of optimum solutions, we present a new Robust Multi-Objective Genetic Algorithm (RMOGA) that optimizes two objectives: a fitness value and a robustness index. The fitness value serves as a measure of performance of design solutions with respect to multiple objectives and feasibility of the original optimization problem. The robustness index, which is based on a non-gradient based parameter sensitivity estimation approach, is a measure that quantitatively evaluates the robustness of design solutions. RMOGA does not require a presumed probability distribution of uncontrollable parameters and also does not utilize the gradient information of these parameters. Three distance metrics are used to obtain the robustness index and robust solutions. To illustrate its application, RMOGA is applied to two well-studied engineering design problems from the literature.
\end{abstract}

\section{Categories and Subject Descriptors}

G.1.6 Optimization-Nonlinear Programming;

\section{General Terms}

Design, algorithms

\section{Keywords}

Multi-objective genetic algorithms, robust design optimization, performance and robustness trade-off

Permission to make digital or hard copies of all or part of this work for personal or classroom use is granted without fee provided that copies are not made or distributed for profit or commercial advantage and that copies bear this notice and the full citation on the first page. To copy otherwise, or republish, to post on servers or to redistribute to lists, requires prior specific permission and/or a fee.

GECCO'05, June 25-29, 2005, Washington, DC, USA.

Copyright 2005 ACM 1-59593-010-8/05/0006 ..\$5.00.

\section{INTRODUCTION}

There are many engineering optimization problems in the real world that have parameters with uncontrollable variations due to noise or uncertainty. These variations can significantly degrade the performance of optimum solutions and can even change the feasibility of obtained solutions. The implications of such variations are more serious in engineering design problems that often have a bounded feasible domain and/or where the optimum solutions lie on the boundary of the feasible domain.

Many methods and approaches have been proposed in the literature to obtain robust design solutions; that is, feasible design alternatives that are optimum in their objectives and whose objective performance or feasibility (or both) is insensitive to the parameter variations. Generally, those approaches can be classified into two types: stochastic approaches and deterministic approaches. Stochastic approaches use the probability information of the variable parameters, i.e., their expected value and variance, to minimize the sensitivity of solutions (e.g., Parkinson et al. [1], Yu and Ishii [2], Jung and Lee [3] for objective robust optimization; and Du and Chen [4], Chen et al. [5], Tu, Choi and Park [6,7], Youn et al. [8] and Ray [9] for feasibility robust optimization also called reliability optimization. Also, Jin and Sendhoff [10] proposed an evolutionary approach to deal with the trade-off between performance and robustness using variance information). The main shortcoming of stochastic approaches is that the probability distribution for the uncontrollable parameters is known or presumed. However, it is difficult (or even impossible) to obtain such information beforehand in real-world engineering design problems.

Deterministic approaches, on the other hand, obtain robust optimum design solutions using gradient information of the parameters (e.g., Balling et al. [11], Sundaresan et al. [12, 13], Zhu and Ting [14], Lee and Park [15], Su and Renaud [16], Messac and Yahaya [17]) or using a non-gradient based parameter sensitivity estimation (Gunawan and Azarm [18-21]). The aim of the Gunawan and Azarm's approach [18-21] is to obtain optimum solutions which essentially satisfy an additional robustness constraint that is prescribed by the decision maker (DM).

In this paper, we present a new deterministic approach to investigate the trade-off between the performance and robustness 
of optimum solutions, based on a Multi-Objective Genetic Algorithm (MOGA). The goal of our approach is to simultaneously optimize: i) a measure of the optimum solutions' performance, i.e., the fitness value, that accounts for objective and constraint values in the original optimization problem (defined in Section 2), and ii) a measure of the optimum solutions' robustness, the robustness index, originally proposed by Gunawan and Azarm [18-21], extended in this paper with the use of two additional distance norms. This approach is a deterministic method using non-gradient based parameter sensitivity estimation, which can be applied to optimization problems having objective and/or constraint functions that are non-differentiable with respect to the parameters. Any MOGA in the literature can be applied to this approach.

In Gunawan and Azarm's approach [18-21], the authors tried to obtain optimum solutions that are insensitive to the parameter variation. In other words, the robustness requirement was considered as a constraint in their approach. On the contrary we treat "robustness" as one of our objectives and form a new two-objective robust optimization problem (regardless of how many objectives the original problem has), to investigate the relation between the performance and robustness of solutions. The RMOGA here aims at simultaneously maximizing performance and maximizing robustness.

The organization of the rest of this paper is as follows: In Section 2 , we present the original optimization problem and explain some definitions and terminologies. Based on a brief description of the objective and feasibility robust optimization approach, we present our new approach in Section 3. Section 4 demonstrates the application of our approach to two test problems followed by a discussion of the results. The paper concludes with a summary in Section 5.

\section{PROBLEM DEFINITION}

In this section, we formally define the problem and explain several definitions and terminologies used in this paper.

A general formulation of multi-objective optimization problem is shown in (1).

$$
\begin{array}{ll}
\min _{x} & f_{m}(x, p) \quad m=1, \ldots, M \\
\text { s.t. } & g_{j}(x, p) \leq 0 \quad j=1, \ldots, J \\
& x^{\text {lower }} \leq x \leq x^{\text {upper }}
\end{array}
$$

$\left(f_{1}, \ldots, f_{M}\right)^{\mathrm{t}}$ are the objective functions (' $\mathrm{t}$ ' refers to a transpose of the row vector), $x=\left(x_{1}, \ldots, x_{N}\right)^{\mathrm{t}}$ is the design variable vector (controlled in an optimization run), $p=\left(p_{1} \ldots, p_{G}\right)^{\mathrm{t}}$ is the uncontrollable parameter vector. Note that design variables that themselves have uncontrollable variations are included in both $x$ and $p . \quad x^{\text {lower }}$ and $x^{u p p e r}$ are the lower and upper bounds of $x$, respectively. The problem has $J$ inequality constraints, $g_{j}, j=1, \ldots, J$. We presume that all constraints can be represented as inequality functions. In this paper, we call the optimization problem shown in (1) as the original problem.

Since there are trade-offs amongst the $M$ objectives, usually the original problem has more than one optimum solution. The set of these optimum solutions is called the Pareto set, as discussed in Miettinen [22] and in Deb [23].

In the following, we briefly describe some terminologies that are used in this paper.

Nominal parameter value $p_{0}=\left(p_{0,1}, \ldots, p_{0, G}\right)^{\mathrm{t}}$ is the parameter vector values, $p=p_{0}$, used to optimize the problem in (1). The parameter's variation is $\Delta p=\left(\Delta p_{1}, \ldots, \Delta p_{G}\right)^{\mathrm{t}}$.
Nominal Pareto solutions are the Pareto solutions of the optimization problem in (1) when $p=p_{0}$.

Let $x_{0}$ be a design solution whose robustness we want to analyze. $f\left(x_{0}, p_{0}\right)=\left(f_{l}\left(x_{0}, p_{0}\right), \ldots, f_{M}\left(x_{0}, p_{0}\right)\right)$ are the nominal values for the objective functions, and $g\left(x_{0}, p_{0}\right)=\left(g_{1}\left(x_{0}, p_{0}\right), \ldots, g_{J}\left(x_{0}, p_{0}\right)\right)$ are the nominal values for the constraint functions.

Tolerance Region is a hyper-rectangular region in $\Delta p$-space formed by a set of $\Delta p$ values with respect to which the decision maker wants the robust optimum solution to be insensitive. This region is usually bounded by $\Delta p^{\text {lower }} \leq \Delta p \leq \Delta p^{\text {upper }}$, where the known $\Delta p^{\text {lower }}$ and $\Delta p^{\text {upper }}$ are the lower and upper bounds of $\Delta p$, respectively, $\quad \Delta p^{\text {lower }}=\left(\Delta p_{1}^{\text {lower }}, \ldots, \Delta p_{G}^{\text {lower }}\right)^{\mathrm{t}} \quad$ and $\Delta p^{\text {upper }}=\left(\Delta p_{1}{ }^{\text {upper }}, \ldots, \Delta p_{G}{ }^{\text {upper }}\right)^{\mathrm{t}}$. For simplicity, the tolerance region is assumed to be symmetric, i.e., $-\Delta p_{i}^{\text {lower }}=\Delta p_{T, i}=\Delta p_{i}^{\text {upper }}$, $\Delta p_{T, i}>0 \forall i=1, \ldots, G$. Since there can be more than one uncontrollable parameter with different magnitudes, we normalize the tolerance region to form a hyper-square.

Parameter variation space ( $\Delta p$-space): A G-dimensional space in which the axes are the parameter variation $\Delta p$ values.

Acceptable Performance Variation Region (APVR) is the region formed in the objective function space around the point $f\left(x_{0}, p_{0}\right)$, which represents the maximum acceptable performance variation chosen by the DM, i.e., $\Delta f_{0}=\left(\Delta f_{0,1}, \ldots, \Delta f_{0, M}\right)^{\mathrm{t}}$, where $\Delta f_{0, i} \geq 0, \forall i=1, \ldots, M$. See Figure 1(a) for details.

Fitness value $f_{v}$ is a value that measures a solution's performance in a combined objective and constraint sense. The fitness value (or rank) obtained from a MOGA, e.g., NSGA [23], can be used as the fitness value in our approach.

Robustness index $\eta$ is a ratio that calculates the radius of the worst-case sensitivity region (defined in Sec. 3.2) with respect to $\Delta p$ over the radius of the exterior hyper-sphere of normalized tolerance region [18]. It is used as a robustness measure in our method. We will discuss it further in Section 3.

Distance norm $\mathrm{L}_{\mathrm{p}}$ for an $N$-dimensional vector $x$ is the vector norm $|x|_{p}$ for $p=1,2$ and infinity $(\infty)$ defined as $|x|_{p}=\left(\sum_{i=1}^{N}\left|x_{i}\right|^{p}\right)^{\frac{1}{p}}$. The infinity norm is: $|x|_{\infty} \equiv \max _{i}\left|x_{i}\right|$.

\section{ROBUST MOGA}

In this section, we first briefly discuss the sensitivity estimation proposed by Gunawan and Azarm [18-21], based on which, the measures for robustness and performance of solutions are defined. We then present our two-objective RMOGA approach.

\subsection{Parameter Sensitivity Estimation}

We first discuss the approach for multi-objective robust optimization, followed by the approach for feasibility robust optimization and then the combined approach.

Given an APVR for a solution $x_{0}$, there is a set of $\Delta p$ such that the variation in objective functions values due to the $\Delta p$ are still within the ranges of $\Delta f_{0, i}$ for all $i=1, \ldots, M$. This set of $\Delta p$ forms a hyper-region around the origin in $\Delta p$-space, which is called the Sensitivity Region (SR). The region is bounded as shown in (2):

$$
\begin{aligned}
& \left|\Delta f_{i}\right| \leq \Delta f_{0, i}, \forall i=1, \ldots, M \\
& \text { where } \quad \Delta f_{i}=f_{i}\left(x_{0}, p_{0}+\Delta p\right)-f_{i}\left(x_{0}, p_{0}\right)
\end{aligned}
$$

Figure 1 shows the APVR and its corresponding SR for a solution $x_{0}$ in a two- parameter and two-objective case. Graphically, the 
points inside, outside, and on the boundary of the APVR correspond to the points inside, outside, and on the boundary of the SR (Figure 1(b)), respectively.

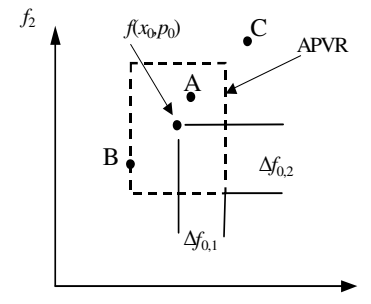

(a)

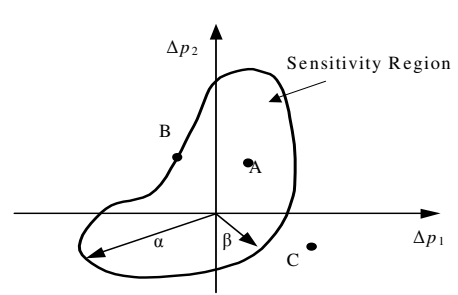

(b)
Figure 1 (a) The APVR and (b) the SR

Essentially, the SR represents the amount of parameter variations that a solution $x_{0}$ can absorb before it violates the APVR. We can use the size of the SR as a measure for the sensitivity of a design: the larger the SR for a design, the more robust that design is. However, in general, the shape of the SR can be asymmetric, which means a design can be very sensitive (or much less robust) in a certain direction of $\Delta p$ (such as direction $\beta$ in Figure 1(b)), but much less sensitive (or very robust) in other directions (such as direction $\alpha$ in Figure 1(b)). To overcome this asymmetry, a Worst-Case Sensitivity Region (WCSR) is used to estimate the SR of a design. The WCSR is a symmetric hyper-sphere that approximates the SR. Graphically, the WCSR is the smallest hyper-sphere that touches the SR at the closest point to the origin, as shown in Figure 2 for a two-parameter case.

Since the WCSR is symmetric, the radius of the WCSR, $R_{f}$, instead of the size of the WCSR, could be used as our robustness measure. It measures the overall robustness of a design. The radius of the WCSR for $x_{0}$ can be calculated by solving a single-objective optimization problem shown in (3).

$$
\begin{array}{ll}
\min _{\Delta p} & R_{f}(\Delta p)=\left[\sum_{j}^{G}\left|\Delta p_{j}\right|^{q}\right]^{\frac{1}{q}} \\
\text { s.t. } \quad \max _{i=1, \ldots, M}\left(\frac{\left|\Delta f_{i}\right|}{\Delta f_{0, i}}\right)-1=0 \\
\text { where } \quad \Delta f_{i}=f_{i}\left(x_{0}, p_{0}+\Delta p\right)-f_{i}\left(x_{0}, p_{0}\right)
\end{array}
$$

In this problem, the design variables are the $\Delta p$ 's, the objective function is the radius of the WCSR. The equality constraint function means that the resultant vector of $\Delta f_{i}$ touches the boundary of the APVR, which means that $\Delta p$ is on the boundary of the sensitivity region. Detailed discussion of this WCSR estimation approach is given elsewhere $[18,19]$.

A similar approach can be used for the feasibility robust optimization. For a design $x_{0}$, all $\Delta p$ points whose corresponding constraint function value $g_{j}\left(x_{0}, p_{0}+\Delta p\right) \leq 0, j=1, \ldots, J$, form the Feasibility Sensitivity Region (FSR), which means the $\Delta p$ inside the FSR will not change the feasibility of design $x_{0}$. The Feasibility WCSR (or FWCSR) is the worst-case estimate of the FSR (similar to the WCSR) and $R_{g}$ is the radius of the normalized FWCSR. $R_{g}$ can be calculated by (4).

$$
\begin{aligned}
& \min _{\Delta p} R_{g}(\Delta p)=\left[\sum_{j}^{G}\left|\Delta p_{j}\right|^{q}\right]^{\frac{1}{q}} \\
& \text { s.t. } \max _{j=1, \ldots, J}\left[g_{j}\left(x_{0}, p_{0}+\Delta p\right)\right]=0
\end{aligned}
$$

Since the SR and the FSR are defined in the same $\Delta p$-space and are of the same scale, $R=\min \left(R_{f}, R_{g}\right)$ implies that we are looking for the radius of worst-case estimate of the intersection of the SR and the FSR for a design solution, as shown in Figure 2.

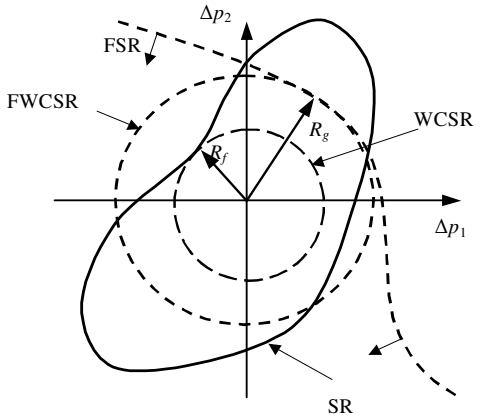

Figure 2 The intersection of SR and the FSR

The radius $R$ can be calculated by solving the optimization problem shown in (5).

$$
\begin{aligned}
& \min _{\Delta p} R(\Delta p)=\left[\sum_{j}^{G}\left|\Delta p_{j}\right|^{q}\right]^{\frac{1}{q}} \\
& \text { s.t. } \quad \max \left\{\max _{i=1, \ldots, M}\left(\frac{\left|\Delta f_{i}\right|}{\Delta f_{0, i}}\right), \max _{j=1, \ldots, J}\left(\frac{\Delta g_{j}}{\left|g_{j}\left(x_{0}, p\right)\right|}\right)\right\}-1=0 \\
& \text { where } \\
& \qquad \Delta g_{j}\left(x_{0}, p_{0}+\Delta p\right)=g_{j}\left(x_{0}, p_{0}+\Delta p\right)-g_{j}\left(x_{0}, p_{0}\right), j=1, \ldots, J
\end{aligned}
$$

For example, in the case shown in Figure $2, R=\min \left(R_{f}, R_{g}\right)=R_{f}$.

\subsection{Robustness Index}

The radius $R$ represents a solution's robustness on an ordinal scale and does not have a physical association with the design solution itself. As such it can be difficult for the DM to do the trade-off analysis between the performance and robustness, i.e., given $R$, $\mathrm{s} /$ he can not decide whether a design solution is robust or not. To overcome this difficulty, we use the radius of the exterior hyper-sphere of the normalized tolerance region, $R_{E}$, as a reference for the robustness requirement (Figure 3). We define the robustness index $\eta=\frac{R}{R_{E}}$ and use this robustness index as one of the two objectives in our RMOGA. $R$ is the optimum solution calculated in (5). Since $R_{E}$ is the radius of the exterior circle of the normalized tolerance region, if $\eta=\frac{R}{R_{E}} \geq 1$, then the design $x_{0}$ is robust.

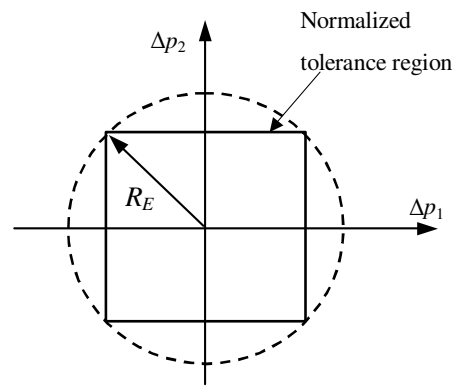

Figure 3 The exterior circle of the normalized tolerance region 


\subsection{Fitness Value}

Recall that our goal in this paper is to simultaneously maximize the performance and robustness of a design. The robustness index serves as a measure of robustness of the design solutions. Hence we need another measure for the overall performance of design solutions.

In multi-objective optimization problem, MOGA is a good tool to obtain Pareto optimum solutions. Most MOGAs assign a fitness value or a rank to each alternative solution in the population to represent its relative goodness, accounting for both objective values and constraints. So the fitness value (or rank ordering) obtained from any MOGA approach, e.g. the rank value from NSGA [23], can be used as the performance measure in our approach: the smaller the fitness value, the better the performance of the solution. For more details on how to obtain this fitness value the reader is referred to [23].

Note that different MOGA approaches may generate different solutions in our approach. However, our goal here is not to develop a new genetic algorithm or distinguish between different MOGAs.

\subsection{RMOGA Approach}

Given the two measures for performance and robustness of a design solution, as discussed before, we can formulate our problem that has two objectives: one is the performance and the other is the robustness for a design solution. The formulation of our robust multi-objective optimization problem is shown in (6)

$$
\begin{array}{ll}
\min _{x} & f_{v}\left(f_{1}, \ldots, f_{M}, g_{1}, \ldots, g_{G}\right) \\
\max _{x} & \eta=\frac{R}{R_{E}}
\end{array}
$$

Here the fitness value $f_{v}$ is a function of the objectives and constraints that are calculated in (1). The robustness index $\eta$ is calculated from (5).

An optimization approach (Figure 4), with an outer-inner structure, is utilized to solve the problem shown in (6). The outer sub-problem (i.e., the upper sub-problem in Figure 4) is to simultaneously minimize the fitness value $f_{v}$ and maximize the robustness index $\eta$. We use the inner sub-problem (i.e., the lower sub-problem in Figure 4) to calculate the radius $R$ (recall (5)) with respect to $\Delta p$.

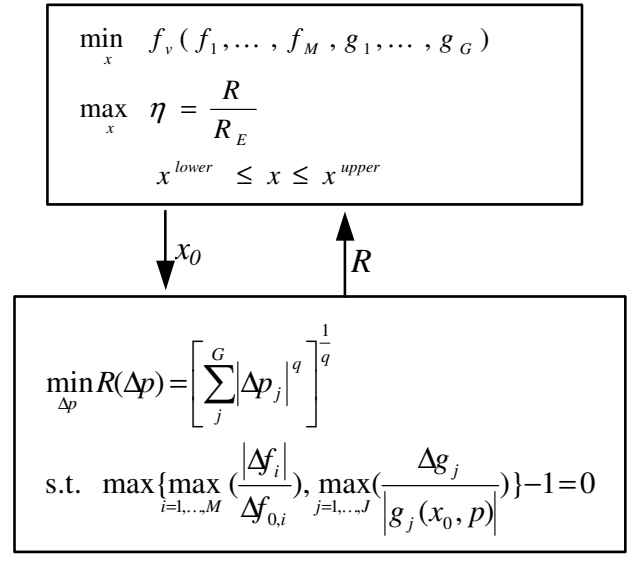

Figure 4 Outer-inner optimization structure of RMOGA
We start with an $x$ value, called $x_{0}$, in the outer sub-problem and send it to the inner sub-problem (including the nominal values for $f_{m}\left(x_{0}, p_{0}\right)$ and $\left.g_{j}\left(x_{0}, p_{0}\right)\right)$. The nominal values are fixed in the inner sub-problem. We then optimize the radius $R$ as a function of $\Delta p$ for the nominal design $x_{0}$, and the optimal value $R$ is sent back to the outer sub-problem. This is repeated for all design alternatives under consideration (see Figure 4).

We now discuss the fitness assignment procedure used in the implementation of RMOGA. For conciseness, the MOGA details are not discussed here and the focus is on the RMOGA. The Genetic Algorithm (GA) requires a scalar fitness value for all the candidate solutions. The major steps in the fitness assignment procedure are as follows:

Step 1: Evaluate the objectives and constraints of the original problem (1).

Step 2: Calculate the robustness index $\eta$ for each of the candidate solutions.

Step 3: Perform a non-dominated sort on the population based on the objective values of the original problem. Consider this rank and the constraint violation to assign a fitness value $f_{v}$ to the candidate solution.

Step 4: Perform a non-dominated sorting on the population based on robust index $\eta$ and fitness value $f_{v}$ as the objectives. This is essentially a two-objective non-dominated sorting.

Step 5: Assign a fitness based on the non-dominated rank from Step 4 and continue the GA iterations until convergence is reached.

\subsection{Distance Norm}

In (5) we could use three different $q$ values $q=1,2$ and $\infty$. Different $\mathrm{L}_{\mathrm{q}}$-norms will affect the value of the robustness index of a design solution. As shown in Figure 5, the distance from point A, B and C to the origin correspond to the radius solution from (5) in $\mathrm{L}_{1}, \mathrm{~L}_{2}$ and $\mathrm{L}_{\infty}$ norm. The robustness measure should be specified in a particular distance-norm.

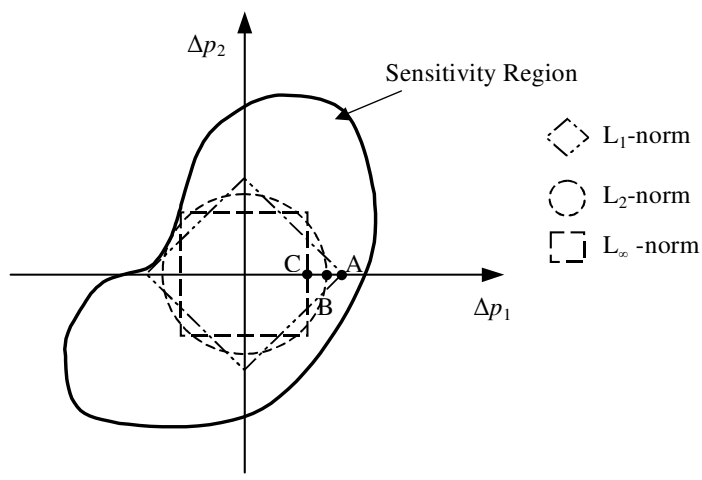

Figure 5 The effect of different $L_{p}$ - norms

\section{TEST RESULTS}

In this section, we will demonstrate an application of the proposed approach to two test problems.

\subsection{Test Problem 1}

\subsubsection{Problem Description}

The first test problem that we use to demonstrate the RMOGA 
approach is a popular test problem from the engineering design optimization literature, also studied by Kirsch [24] and Deb [23].

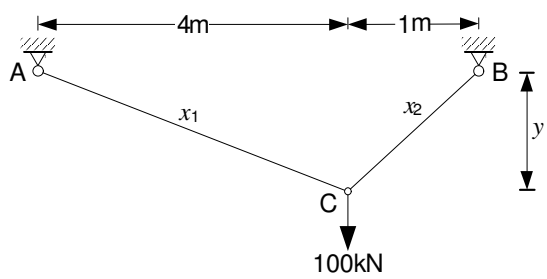

Figure 6 Schematic for two-bar truss design problem

The problem is to design a two-bar truss that can carry a single vertical load of $100 \mathrm{kN}$ at joint $\mathrm{C}$. The truss comprises of two links as shown in the Figure 6 . The objectives are to minimize the volume of the two links and to minimize the stress in them as well. The variables are the cross-sectional areas of the links, $x_{1}, x_{2}$, and the vertical drop of the joint $y$. The constraints are: an upper limit of $100,000 \mathrm{kN} / \mathrm{m}^{2}$ for the stress, the range $1.0-3.0 \mathrm{~m}$ for $y$, and a non-negative value for the cross-sectional areas. The problem is formulated as follows:

$$
\begin{array}{ll}
\min & f_{1}\left(x_{1}, x_{2}, y\right)=x_{1} \sqrt{16+y^{2}}+x_{2} \sqrt{1+y^{2}} \\
\min & f_{2}\left(x_{1}, x_{2}, y\right)=\frac{20 \sqrt{16+y^{2}}}{x_{1} y} \\
\text { s.t. } & \\
& 20\left(16+y^{2}\right)^{1 / 2}-100000 \quad y x_{1} \leq 0 \\
& 80\left(1+y^{2}\right)^{1 / 2}-100000 \quad y x_{2} \leq 0 \\
& 1 \leq y \leq 3 \\
& x_{1}, x_{2} \geq 0
\end{array}
$$

\subsubsection{RMOGA Solutions}

The known variation in the design variables was set as $\Delta x_{1}=\Delta x_{2}=0.0001$ and $\Delta y=0.05$. The acceptable performance variation $\Delta f_{0,1}$ and $\Delta f_{0,2}$ was set to $0.75 \mathrm{kN} / \mathrm{m}^{2}$ for both.

Figure 7 shows the Pareto optimum solutions as well as all other (dominated or inferior) solutions obtained by solving problem (6). It can be seen that of all the solutions with the best fitness value, $f_{v}=1$, only one design is robust, i.e., it has a robustness index $\eta$ greater than 1 . On the other hand we see that several solutions with a lower fitness value ( $f_{v}$ greater than 1$)$ are robust. However, there are only a few Pareto optimum solutions obtained for the problem defined in (6), but these solutions are the best in terms of both robustness and the fitness value of the original problem. It is possible that a decision maker might not choose a solution from this set of Pareto optimum solutions as these solutions might prove more robust than is necessary.

Figure 8 compares the Pareto optimum solutions obtained by RMOGA (i.e., RMOGA Pareto) as shown in (6) and mapped on to the objective function space, with those solutions (i.e., Nominal Pareto) obtained by a traditional MOGA that does not consider robustness. We observe that the Pareto optimum solutions obtained by RMOGA are further away from the origin (compared to Nominal Pareto), which as expected leads us to conclude that the Nominal Pareto solutions obtained by a MOGA are not necessarily robust.

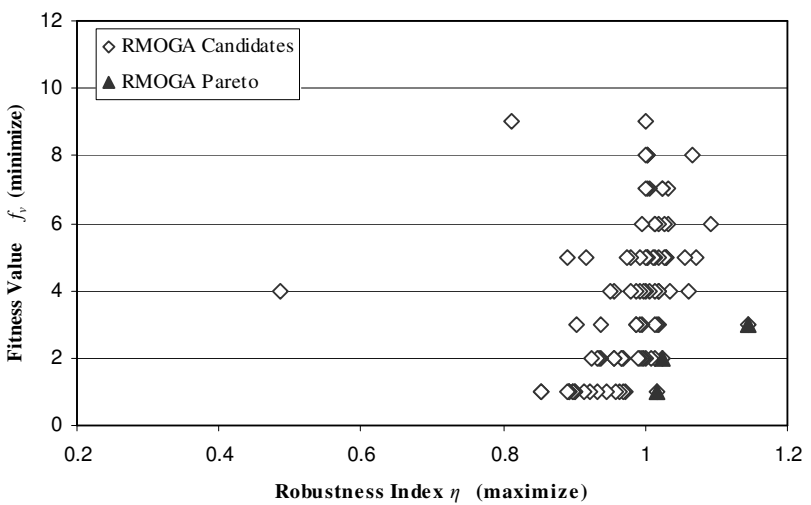

Figure 7 Fitness value vs. robustness index for two-bar truss problem using RMOGA (with $\mathrm{L}_{2}$ norm for $R$ )

Figure 8 also includes all solutions from Figure 7 that have a robustness index of greater than or equal to $1(\eta \geq 1)$ irrespective of their fitness value $f_{v}$.

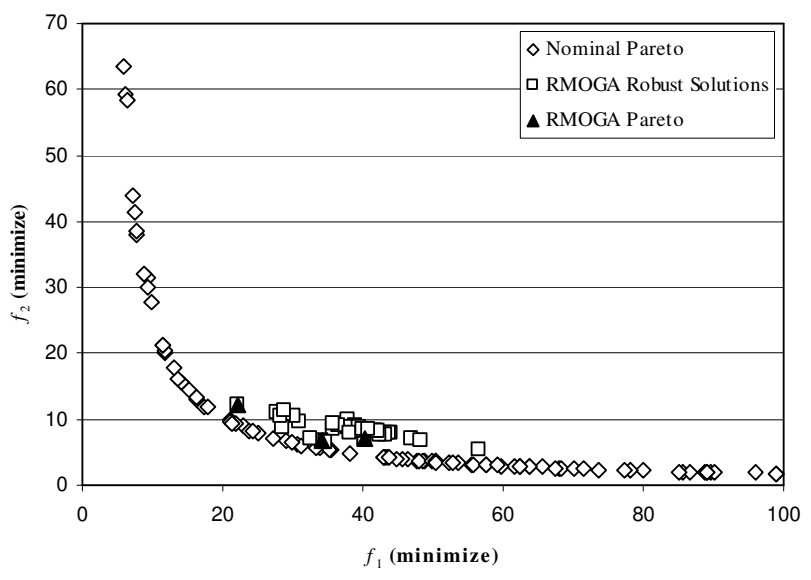

Figure 8 Comparison of Nominal Pareto and RMOGA $\left(\mathrm{L}_{2}\right.$ norm) solutions for the two-bar truss problem

Figure 9 compares the robustness index $\eta$ for the solutions with $f_{v}=1$ (the best fitness value in RMOGA, see Figure 7 with $\mathrm{L}_{2}$ norm) obtained using different distance metrics. As shown in Figure 9, there is some overlap between the solutions with respect to $\eta$.

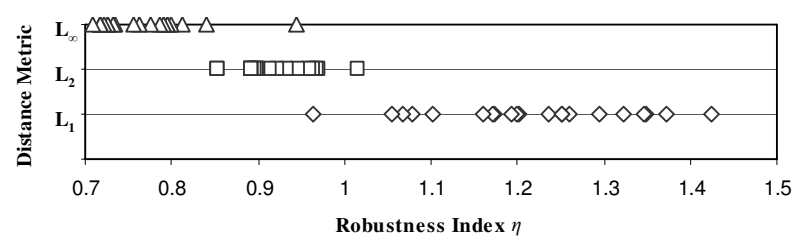

Figure 9 Comparison of robustness index of solutions with $f_{v}=1$ using different distance metrics for the two-bar truss problem 


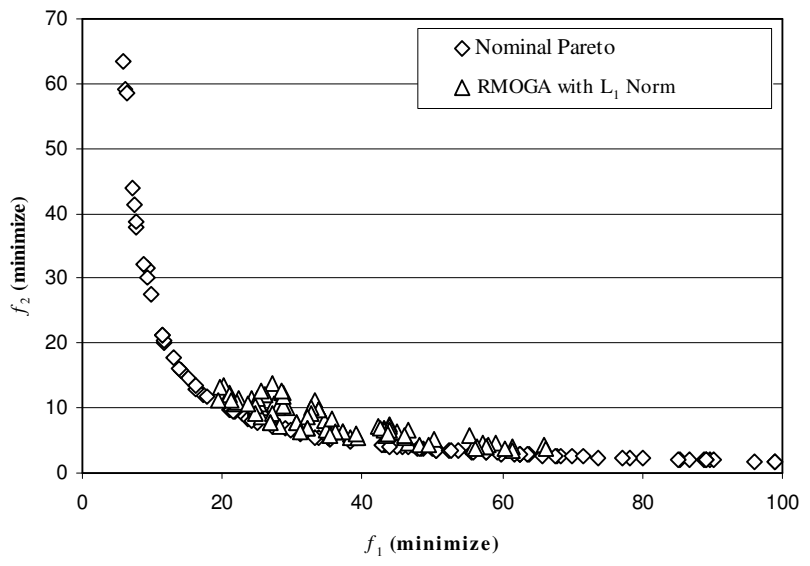

Figure 10 Comparison of Nominal Pareto with robust solutions using RMOGA with $\mathrm{L}_{1}$ norm for the two bar truss problem

Figure 10 compares the Nominal Pareto optimum solutions with all the robust designs obtained from RMOGA using the $\mathrm{L}_{1}$ norm distance metric (RMOGA with $\mathrm{L}_{1}$ norm). Most of the robust solutions obtained using the $\mathrm{L}_{1}$ norm are further away from the origin which implies that the solutions belonging to the Nominal Pareto front are not necessarily robust. Finally, note that no robust ( $\eta \geq 1$ ) solutions were obtained using the $\mathrm{L}_{\infty}$ norm.

From the simulation results for this example (see Figure 8 and Figure 10), we can conclude that the calculated robustness index is largely dependent upon the distance metric used. This also implies that robustness of design solutions obtained using RMOGA is largely dependent on the type of distance metric used to calculate the robustness index.

\subsection{Test Problem 2}

\subsubsection{Problem Description}

The second test problem is to robustly design a simple speed reducer that might be used in a light airplane between the engine and the propeller. The problem is taken from Azarm and Li [25], but slightly revised here to form a multi-objective optimization problem. A schematic of the speed reducer to be optimized is shown in Figure 11. The first design objective is to minimize the volume of the speed reducer and the second objective is to minimize the stress in the first gear shaft. The problem has seven design variables: gear face width $\left(x_{1}\right)$, teeth module $\left(x_{2}\right)$, number of teeth in the pinion $\left(x_{3}\right.$, integer), distance between bearings $1\left(x_{4}\right)$, distance between bearings $2\left(x_{5}\right)$, diameter of shaft $1\left(x_{6}\right)$ and diameter of shaft $2\left(x_{7}\right)$.

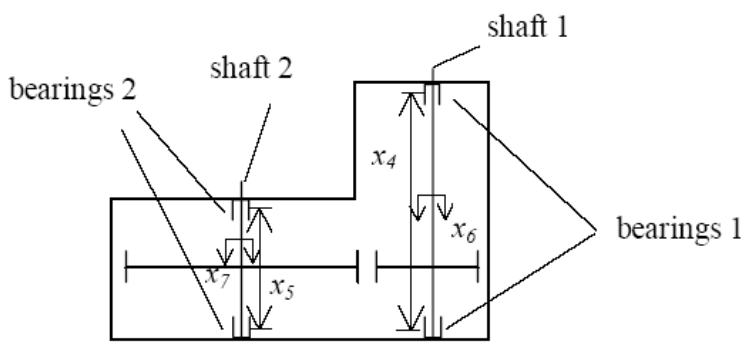

Figure 11 Schematic for speed reducer design problem
The design is subject to a number of constraints imposed by gear and shaft design practices. The seven design variables are subject to an upper and a lower bound. There are eleven inequality constraints that take into consideration: stresses, deflections, space restrictions and design requirements. The units for all the design variables are in cm except for $x_{3}$ and those of the objectives $f_{1}$ and $f_{2}$ are $\mathrm{cm}^{3}$ and $\mathrm{kPa}$, respectively. The formulation of the problem is as follows:

$\min f_{1}=$

$0.7854 \quad x_{1} x_{2}^{2}\left(\begin{array}{lllll}10 & \left.x_{3}^{2} / 3+14.933 x_{3}-43.0934\right)-\end{array}\right.$

$1.508 x_{1}\left(x_{6}^{2}+x_{7}^{2}\right)+7.477\left(x_{6}^{3}+x_{7}^{3}\right)+0.7854\left(x_{4} x_{6}^{2}+x_{5} x_{7}^{2}\right)$

$\min f_{2}=\frac{\sqrt{\left(745 x_{4} / x_{2} x_{3}\right)^{2}+1.69 \times 10^{7}}}{0.1 x_{6}^{3}}$

s.t.

$g_{1}: \frac{1}{\left(x_{1} x_{2}^{2} x_{3}\right)}-\frac{1}{27} \leq 0, \quad g_{2}: \frac{1}{\left(x_{1} x_{2}^{2} x_{3}^{2}\right)}-\frac{1}{397.5} \leq 0$

$g_{3}: \frac{x_{4}^{3}}{\left(x_{2} x_{3} x_{6}^{4}\right)}-\frac{1}{1.93} \leq 0, \quad g_{4}: \frac{x_{5}^{3}}{\left(x_{2} x_{3} x_{7}^{4}\right)}-\frac{1}{1.93} \leq 0$

$g_{5}: x_{2} x_{3}-40 \leq 0, \quad g_{6}: \frac{x_{1}}{x_{2}}-12 \leq 0$

$g_{7}: 5-\frac{x_{1}}{x_{2}} \leq 0, \quad g_{8}: 1.9-x_{4}+1.5 x_{6} \leq 0$

$g_{9}: 1.9-x_{5}+1.1 x_{7} \leq 0, \quad g_{10}: f_{1} \leq 1300$

$g_{11}: \frac{\sqrt{\left(745 x_{5} / x_{2} x_{3}\right)^{2}+1.575 \times 10^{8}}}{0.1 x_{7}^{3}} \leq 1100$

$2.6 \leq x_{1} \leq 3.6 \quad 0.7 \leq x_{2} \leq 0.8$

$17 \leq x_{3} \leq 28 \quad 7.3 \leq x_{4}, x_{5} \leq 8.3$

$2.9 \leq x_{6} \leq 3.9 \quad 5.0 \leq x_{7} \leq 5.5$

\subsubsection{RMOGA Solutions}

The known variation in the design variables $\Delta x_{1}$ and $\Delta x_{2}$ was set as 0.01 and 0.1 , respectively. The corresponding acceptable performance variation $\Delta f_{0,1}$ and $\Delta f_{0,2}$ were set to 225 and 125 , respectively. The solutions for the speed reducer problem obtained from the RMOGA as well as the traditional MOGA are compared and discussed in this section.

From Figure 12, we observe that not many solutions with the best fitness $\left(f_{v}=1\right)$ are robust. Similar results were observed for the test problem 1 as well, based on which we can conclude that the best design solutions in terms of performance (objectives and constraints) are not necessarily robust. The RMOGA problem (6) has only three Pareto optimum solutions as shown in Figure 12. When we map those solutions from Figure 12 into the objective function space, we have the plot shown in Figure 13. There is an overlap (one point) between the RMOGA Pareto solutions and the Nominal Pareto front because this design solution is not robust (in $\mathrm{L}_{2}$ norm).

Figure 13 also compares all the robust solutions (i.e., RMOGA robust solutions) as well as the RMOGA Pareto solutions (using $\mathrm{L}_{2}$ norm) with the Nominal Pareto front. Similar to the first test problem, some RMOGA robust solutions are further away from the origin compared to Nominal Pareto solutions. Figure 14 shows a comparison of the robustness index $\eta$ for the solutions with $f_{v}=1$ (the best fitness value in RMOGA, see Figure 12 for $\mathrm{L}_{2}$ norm) obtained using the three different distance metrics. As shown in Figure 14, most of the solutions from $\mathrm{L}_{1}$ and $\mathrm{L}_{\infty}$ norms are robust while none from $\mathrm{L}_{2}$ norm is robust (with $f_{v}=1$ ). Figures 15 and 16 compare the Nominal Pareto solutions with all robust $(\eta \geq 1)$ solutions obtained from RMOGA with $\mathrm{L}_{1}$ and $\mathrm{L}_{\infty}$ norms, respectively. 


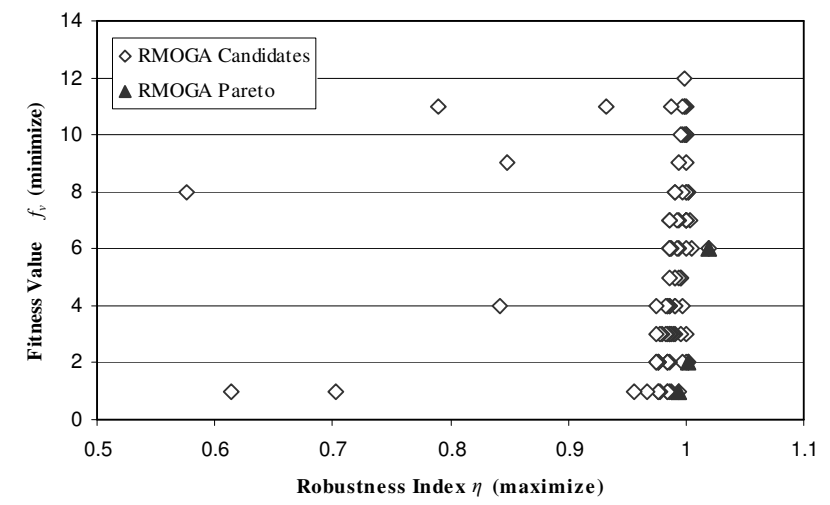

Figure 12 Fitness value vs. robustness index results for the speed reducer problem from RMOGA (with $L_{2}$ norm for $R$ )

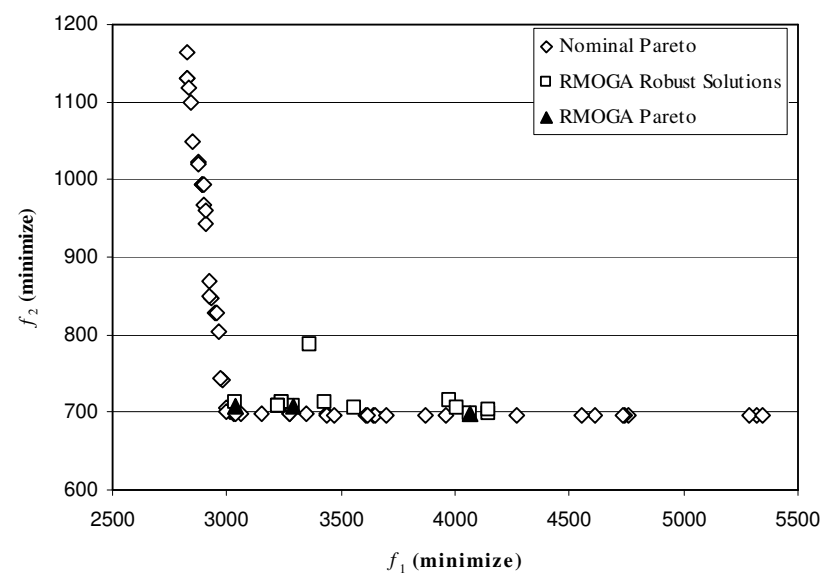

Figure 13 Comparison of Nominal Pareto with RMOGA $\left(\mathbf{L}_{2}\right.$ norm) solutions for speed reducer problem

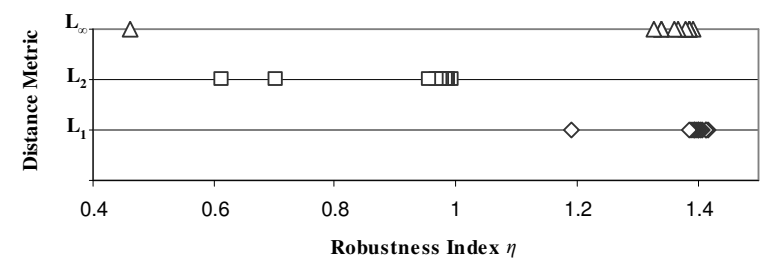

Figure 14 Comparison of robustness index of different solutions with $f_{v}=1$ obtained using different distance metrics for speed reducer problem

For $\mathrm{L}_{1}$ and $\mathrm{L}_{\infty}$ norms as well, we see that the robust solutions are further away from the origin compared to the Nominal Pareto solutions. Comparing the robust solutions in Figure 13, 15 and 16, we conclude that the robustness of design solutions is dependent on different distance metrics used to calculate the radius $R$ in (5). And this implies that, as in test problem 1, robustness of design solutions obtained using RMOGA is largely dependent on the type of distance metric used to calculate the robustness index.

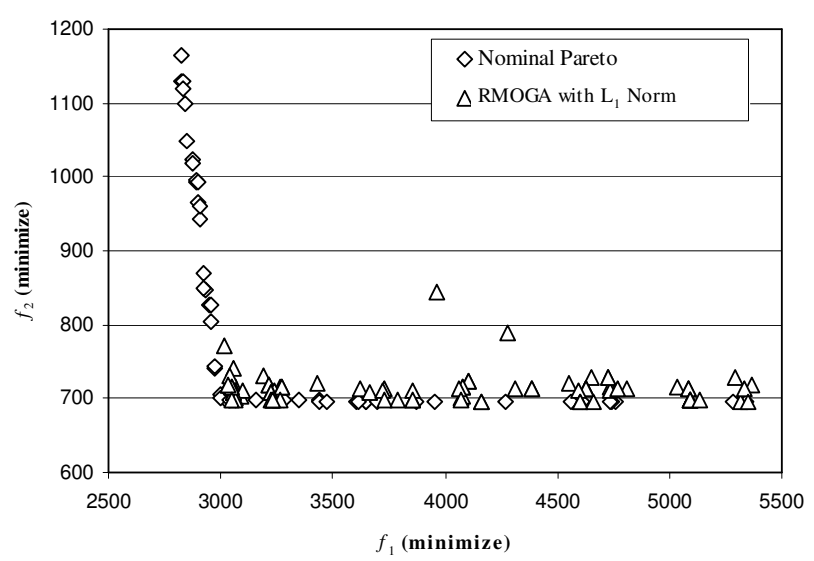

Figure 15 Comparison of Nominal Pareto solutions with robust solutions obtained using RMOGA with $\mathrm{L}_{1}$ norm for speed reducer problem

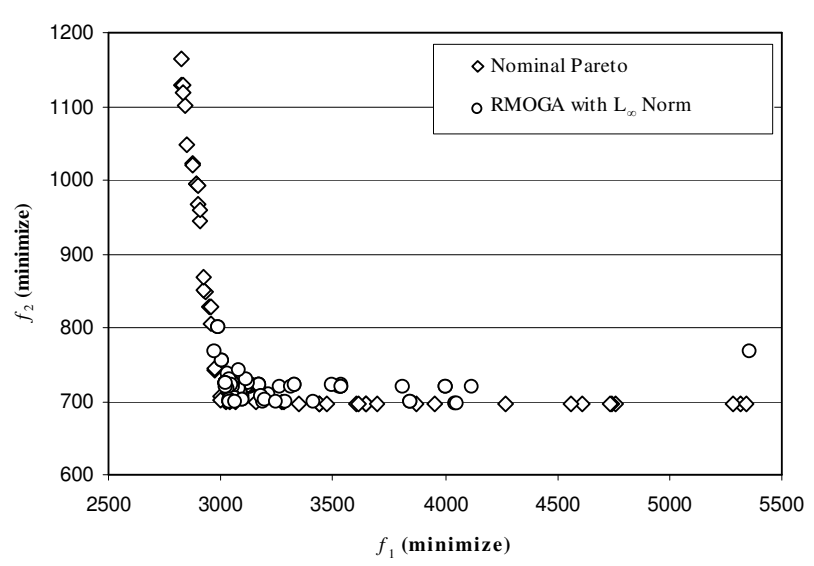

Figure 16 Comparison of Nominal Pareto with robust solutions obtained using RMOGA with $\mathrm{L}_{\infty}$ norm for speed reducer problem

\section{CONCLUSIONS}

A deterministic Robust Multi-Objective Genetic Algorithm (RMOGA) is presented in this paper. This RMOGA gives a set of solutions that are Pareto optimum in terms of the performance (i.e., minimization of fitness value) and the robustness (i.e., maximization of robustness index). The fitness value accounts for the objectives and constraints of the original problem. The robustness index also accounts for variations in the objectives as well as constraints. The trade-off between the performance and robustness of a design solution has been shown. RMOGA uses an outer-inner optimization structure to solve the overall problem. Both the outer and inner optimization sub-problems are solved using a binary coded genetic algorithm. Any MOGA can be used in our approach. The approach does not require a presumed probability distribution of uncontrollable parameters and also does not utilize the gradient information of these parameters. Three different Euclidian distance metrics are used in conjunction with RMOGA to calculate the robustness index.

This approach is applied to two engineering test problems. The results from RMOGA are compared to Nominal Pareto solutions. The robust design solutions obtained from RMOGA are 
marginally inferior to the Nominal Pareto solutions in terms of performance. But at the same time, they are least sensitive to the variation in design parameters. In the two test problems it was found that the best design solutions in terms of performance (objectives and constraints) are not necessarily robust. RMOGA brings to light this trade-off between the performance of a design solution and its robustness, and thus can assist the DM in choosing the best possible solution which is also robust. Based on the simulation results, we conclude that the robustness of designs is largely dependent on the type of distance metric used to calculate the robustness index.

\section{ACKNOWLEDGMENTS}

The work presented in this paper was supported in part by a contract from Indian Head Division, NSWC. Indian Head Division was funded by the US Office of Naval Research. The work was also supported in part by the National Science Foundation through Grant DMI 200029. Such support does not constitute an endorsement by the funding agency of the opinions expressed in the paper.

\section{REFERENCES}

[1] Parkinson, A., Sorensen. C. and Pourhassan, N. A., 1993, "General Approach to Robust Optimal Design," Trans. ASME, Journal of Mechanical Design, 115, 74-80.

[2] Yu, J.C. and Ishii, K., 1998, "Design for Robustness Based on Manufacturing Variation Patterns." Trans. ASME, Journal of Mechanical Design, 120, 196 - 202.

[3] Jung, D. H. and Lee, B. C., 2002, "Development of a Simple and Efficient Method for Robust Optimization," Int. Journal for Numerical Methods in Engineering, 23, 2201-2215.

[4] Du, X., and Chen, W., 2000, "Towards a Better Understanding of Modeling Feasibility Robustness in Engineering Design," Trans. ASME, Journal of Mechanical Design, 122, 385-394.

[5] Chen, W., Wiecek, M.M. and Zhang, J., 1999, "Quality Utility - A Compromise Programming Approach to Robust Design." Trans. ASME, Journal of Mechanical Design, 121, 179-187.

[6] Tu, J., Choi, K. K. and Park, Y. H., 1999, “A New Study on Reliability-Based Design Optimization," Trans. ASME, Journal of Mechanical Design, 121, 557-564.

[7] Choi, K K., Tu, J. and Park, Y. H., 2001, "Extensions of Design Potential Concept for Reliability-Based Design Optimization to Nonsmooth and Extreme Cases," Structural and Multidisciplinary Optimization, 22, 335-350.

[8] Youn, B. D., Choi, K. K. and Park, Y. H., 2003, "Hybrid Analysis Method for Reliability-Based Design Optimization," Trans. ASME, Journal of Mechanical Design, 125, 221-232.

[9] Ray, T., 2002, "Constrained Robust Optimal Design using a Multiobjective Evolutionary Algorithm," in Congress on Evolutionary Computation (CEC'2002), 1, 419-424, IEEE Service Center, Piscataway, New Jersey.

[10] Jin, Y. and Sendhoff, B., 2003, "Trade-Off between Performance and Robustness: An Evolutionary Multiobjective Approach," Evolutionary Multi-Criterion
Optimization. Second International Conference, EMO 2003, 237-251, Springer. Lecture Notes in Computer Science. 2632, Faro, Portugal.

[11] Balling, R. J., Free, J. C. and Parkinson, A. R., 1986, "Consideration of Worst-Case Manufacturing Tolerances in Design Optimization," Trans. ASME, Journal of Mechanical Design, 108, 438-441.

[12] Sundaresan. S., Ishii. K. and Houser, D., 1992, "Design Optimization for Robustness Using Performance Simulation Programs," Engineering Optimization, 20, 163-178.

[13] Sunderasan, S., Ishii, K., and Houser, D. R., 1993, “A Robust Optimization Procedure with Variation on Design Variables and Constraints," Advances in Design Automation, 65, No. 1, 379-386.

[14] Zhu, J. and Ting, K. L., 2001, "Performance Distribution Analysis and Robust Design." Trans. ASME, Journal of Mechanical Design, 123, 11-17.

[15] Lee, K. and Park, G., 2001, "Robust Optimization Considering Tolerances of Design Variables," Computers and Structures, 79, 77-86.

[16] Su, J., and Renaud, J. E., 1997, "Automatic Differentiation in Robust Optimization," AIAA, 35, 1072-1079.

[17] Messac, A. and Yahaya, A. I., 2002, "Multiobjective Robust Design Using Physical Programming," Structural and Multidisciplinary Optimization, 23, No. 5, 357-371.

[18] Gunawan, S. and Azarm, S., 2004, "Non-gradient Based Parameter Sensitivity Estimation for Single Objective Robust Design Optimization." Trans. ASME, Journal of Mechanical Design, 126, No.3, 395-402.

[19] Gunawan, S. and Azarm, S., 2005, "Multi-Objective Robust Optimization Using a Sensitivity Region Concept." Structural and Multidisciplinary Optimization, 29(1), 50-60.

[20] Gunawan, S. and Azarm, S., "An Efficient Feasibility Robust Optimization Method using a Sensitivity Region Concept." Trans. ASME, Journal of Mechanical Design, (forthcoming).

[21] Gunawan, S. and Azarm, S., 2004, "On a Combined Multi-Objective and Feasibility Robustness Method for Design Optimization," CD-ROM Proceedings of 10th AIAA/ISSMO MDO, Albany, New York, September.

[22] Miettinen K. M., 1999, Nonlinear Multiobjective Optimization, Kluwer Academic Publishers, Boston.

[23] Deb, K., 2001, Multiobjective optimization using evolutionary algorithms, John Wiley and Sons, New York.

[24] Kirsch, U., 1981, Optimal Structural Design, McGraw-Hill, New York.

[25] Azarm, S. and Li, W.-C., 1990, "Optimality and Constrained Derivatives in Two-Level Design Optimization”, Trans. ASME, Journal of Mechanical Design, 112, 563-568. 